\title{
La excelencia educativa coreana: un modelo en transición
}

$\mathrm{C}$ orea del Sur ha sido reconocida como una nación exitosa en el campo económico, sobre todo por el gran desempeño que han mostrado varias empresas coreanas a nivel internacional. Tal éxito ha sido explicado en parte por la intervención del gobierno y sus políticas de desarrollo. Este trabajo se concentra en la relevancia (o en la falta de ésta) que ha tenido el gobierno en sus esfuerzos por coordinar y orientar el desarrollo educativo del país, de tal manera que favorezca los intereses de competencia empresarial internacional.

A pesar de haberse convertido en una potencia económica junto con los demás tigres del sureste asiático, la educación vocacional y técnica no había sido primordial en el proceso de este avance. Aun en la actualidad se pueden observar tales discrepancias debido al constante desacuerdo entre los intereses del gobierno y las grandes empresas, en contraste con las preferencias que el público muestra en la educación de la juventud.

Según Seth (2002), el desarrollo educativo coreano ha sido tradicionalmente independiente de las necesidades y estrategias económicas del país. Esto no ha sido por falta de esfuerzos gubernamentales hacia una mayor congruencia entre los intereses económicos de la nación y el desarrollo de la educación formal. Por el contrario, desde la desocupación del imperio japonés en la primera mitad del siglo XX, administraciones sucesivas, incluyendo el gobierno militar estadounidense (19451948), se han esforzado por coordinar el desarrollo educativo nacional con los planes económicos. A pesar de dichos esfuerzos, el apoyo hacia la educación vocacional y técnica se ha mostrado en conflicto con la percepción

* Estudiante del Doctorado de Educación Comparada, Universidad Estatal de Ohio, Columbus, Ohio, EUA. del público sobre el propósito de la educación formal.

Para los coreanos, la educación es tradicionalmente un medio crucial por el cual los individuos y sus familias pueden aspirar a adquirir reconocimiento social. Por consiguiente, hay una marcada preferencia en invertir en la educación de la progenie con el objetivo de alcanzar un alto nivel intelectual y esto pocas veces es motivo para preferir una educación técnica o comercial. Paik Hyun-ki escribió en 1968 que la educación fue convertida en un instrumento de avance hacia expectativas personales, por lo que se descuida la función educativa de preparar a las jóvenes generaciones y engendrar en ellas ciudadanos con conciencia comunitaria a través de la cooperación en sociedad. ${ }^{1}$

Durante los años de la ocupación estadounidense, los oficiales gubernamentales, en conjunto con la prensa, hicieron gran hincapié en la creación de escuelas secundarias orientadas a la educación vocacional. Posteriormente, la guerra de las coreas (1950-1953) tuvo como una de sus grandes consecuencias la necesidad de reafirmar las habilidades técnicas indispensables para la reconstrucción nacional. Entonces, maestros y oficiales insistieron en la urgencia de eliminar la tradición confuciana de menospreciar los trabajos que requieren fuerza física. Existía también una gran preocupación por parte de oficiales e intelectuales sobre el desempleo de gente educada. En ese tiempo, tanto los políticos en el gobierno como los líderes de oposición apoyaban en forma unánime esta orientación educativa que, a pesar de todo, era poco popular.

En los años sesenta, bajo el gobierno de Park Chung Hee, renovados esfuerzos por una industrialización fueron evidentes. El compro- 
miso del gobierno de Park hacia un desarrollo económico resultó en un boom económico que impulsó el crecimiento del PIB, con un promedio de 9\% entre 1965 y 1978, comparado sólo con Taiwan y Japón. La administración de Park tenía como principal motivación el fortalecimiento del Estado a través de la sumisión y unificación del pueblo y la expansión de la economía nacional. En otras áreas, como el desarrollo social y las relaciones exteriores, las políticas fueron formuladas de acuerdo con los objetivos económicos o simplemente fueron relegadas a un segundo plano. La educación fue una parte esencial de esta estrategia: el gobierno de Park buscó desde un principio la coordinación de las políticas educativas con la estrategia económica. Entre los años 1960 y 1970 hubo un cambio de la educación liberal hacia la educación vocacional y técnica; una parte de tales esfuerzos fue el establecimiento de cuotas máximas de ingreso a la educación superior.

Sin embargo, mientras que los encargados de aplicar las nuevas políticas educativas utilizaban el entusiasmo del público hacia la educación para transferir la carga financiera sobre el gobierno a los estudiantes y sus familias, y por consiguiente liberar al Estado de la gran inversión que la educación necesitaba, esta misma iniciativa dificultó los esfuerzos por coordinar la educación con la planeación económica. Las administraciones de Park Chung Hee y de Chun Doo Hwan (1980-1988) se caracterizaron por ser autoritarias, pero esto no significa que fueran totalmente independientes del apoyo público. El Estado no estaba aislado de las presiones de los grupos de interés pertenecientes a las clases medias y altas que seguían buscando una educación que favoreciera sus aspiraciones hacia un avance social y de status. Como resultado, el compromiso entre las razones económicas del Estado y la demanda pública por determinada calidad educativa no cambió significativamente.

El primer plan quinquenal de desarrollo económico (1962-1966) puso como objetivo un crecimiento anual del PIB a un 7.1\%, y, como ya se mencionó, éste fue superado. Asimismo, se buscó un aumento en la producción industrial del $15 \%$ y una disminución del desempleo de $24 \%$ a $15 \%$. Industrias como la acerera fueron creadas, y la exportación de textiles, entre otras, creció de manera significativa. El plan gubernamental excedió casi todos sus objetivos. En el área de educación fueron tomadas varias medidas drásticas, como la disminución en cantidad y calidad de programas de humanidades, artes, ciencias sociales, leyes, periodismo, mientras que las áreas técnicas fueron reforzadas.

Entre otras iniciativas también se incluye la creación de las políticas de promoción de educación vocacional, las cuales fueron orientadas al reentrenamiento de profesores, aumentar las materias vocacionales y también las horas mínimas enfocadas a estas materias en todos los niveles escolares. Incluso, se propuso la creación de un plan en el que los años escolares tendrían diferente repartición para favorecer la educación técnica. Dicho plan encontró la oposición de educadores y de padres de familia, escuelas secundarias privadas y colegios, pues vieron en esta propuesta una amenaza a la oportunidad igualitaria de acceso a una educación de calidad y, como resultado, el sistema educativo siguió la tradicional repartición de años en cada nivel (6-3-3-4), similar al mexicano.

Ya que planes como el anterior eran constantemente bloqueados por la opinión pública, la educación vocacional tuvo el riesgo de convertirse en un nuevo medio para la adquisición de una educación puramente académica.

Durante años el problema mayor que afectó la implementación de programas de educación vocacional fue la resistencia pública. Sin embargo, también fue evidente la tendencia de las empresas en contratar egresados universitarios, independientemente de que fueran técnicos o graduados en algún área vocacional, pues las habilidades necesarias para la mayor parte de estos trabajos se podían adquirir en la marcha. Asimismo, los estudiantes inscritos en programas vocacionales y técnicos no perdían la esperanza de continuar en programas académicos una vez finalizados sus estudios vocacio- 
nales. Las regulaciones gubernamentales siguieron apoyando un entrenamiento práctico intensivo, lo que desencadenó un rechazo mayor por parte del público.

Con referencia al significativo aumento en la productividad en los años de Park Chung Hee y posteriores, Cole (1975) mencionó que aparte de la remarcable capacidad laboral que se deriva del sistema educativo (coreano), la fuerza trabajadora coreana tiene otros atributos que han reinformado su contribución al crecimiento económico. Los trabajadores coreanos han sido descritos como un poder humano que es adaptable, capaz de ser entrenado, manualmente hábil y acostumbrado a un arduo trabajo durante largas horas. ${ }^{2}$

Adams y Gottlieb mencionan, asimismo, que la contribución directa de la educación hacia el crecimiento económico se puede ver a través del mejoramiento de habilidades y capacidades productivas e, indirectamente, por medio de una disminución en la fertilidad; dicha afirmación es ampliamente aceptada por intelectuales coreanos.

Los efectos económicos de la inversión en la educación o en el capital humano también son demostrados por medio del remarcado aumento en las tasas de ingreso.

A pesar de la rápida expansión educativa existió también una remarcada competencia en la contratación de graduados universitarios por parte de grandes corporaciones durante los setenta. Sin embargo, la demanda en la educación superior no parece estar relacionada ni tampoco ser la causa de las tasas de participación en la fuerza laboral. En el caso de las mujeres, menos de la mitad de este grupo poblacional eran empleadas des- pués de recibir algún certificado universitario y la incertidumbre general de los beneficios económicos no parecía afectar su desempeño educativo. ${ }^{3}$

La industrialización trajo una nueva estructura ocupacional que fue asociada directamente con el nivel de ingresos y el status social. En un estudio hecho por Adams y Gottlieb fue demostrado que, a pesar de que la diferencia entre regiones no pareció determinar el status social, las riquezas y el nivel educativo han sido factores dominantes en la asignación a determinada clase social tanto en las grandes ciudades como en los suburbios y las áreas rurales. El mismo estudio confirmó que la riqueza personal y el nivel educativo son fuertes determinantes en el nivel de clase social, pero sorpresivamente el nivel de ingresos es un factor relativamente irrelevante. ${ }^{4}$ Estos autores explican que ha existido un cambio entre la importancia que se le da a la riqueza personal o familiar, y que más recientemente, la educación y el tipo de ocupación han aumentado en importancia como referencia al reconocimiento social de los individuos. Esto ha resultado en cambios relevantes en la estructura social por edades y, como consecuencia, en una dominación de las clases medias y las generaciones jóvenes. Este fenómeno es asumido como un producto de la industrialización y el rápido crecimiento educativo.

Los niveles educativos más altos han sido tradicionalmente relacionados con generaciones jóvenes, con un mayor status social y con ocupaciones mejor pagadas. También ha sido evidente una movilidad social intergeneracional, a pesar que las clases sociales más bajas han tendido a continuar sin cambio significativo. ${ }^{5}$ 
Posteriormente, Weidman y Park (2000) identificaron varias perspectivas mediante las cuales diferentes investigadores han explicado la expansión de la educación superior. Tales perspectivas consisten en: el ambiente social, con un enfoque hacia las circunstancias cambiantes del momento; el capital humano, con hincapié en la planeación gubernamental; la radical, que se refiere a la dominación de las clases superiores; la estratificación educativa, remarcando la competencia por status sociales, y la dinámica social, que es dominada por la interacción entre grupos de interés. Dichos autores cuestionaron la validez de estas perspectivas, pues identificaron cierta inclinación parcial en cada una de ellas. En este trabajo pueden ser identificadas varias de ellas; sin embargo, después de una investigación intensiva por parte de dichos autores, a través de entrevistas directas con gente representativa de la estructura de educación superior, emergió una nueva perspectiva ignorada en cualquier otro trabajo previo. Esta perspectiva está enfocada en cada individuo, sin dejar de admitir la interrelación con el sistema.

En este nuevo enfoque, el contexto no puede ser descrito adecuadamente mediante las palabras "lucha" o "competición", más bien es caracterizado por una población involucrada en la guerra por sobrevivir debido a la falta de recursos naturales, la alta densidad poblacional, oportunidades de trabajo inadecuadas y un conflicto constante en contra de las políticas gubernamentales con intención de mantener un control social y económico. Tal guerra por sobrevivir se ha extendido al sector educativo, presionando a la gente hacia intensas batallas por una educación superior.

A partir de la segunda mitad del siglo $\mathrm{XX}$, el sector educativo fue percibido como la única área en la que no existía ningún monopolio; por lo tanto, la certificación educativa se convirtió en un criterio relativamente objetivo y justo en la selección de posiciones laborales. La educación ha sido entendida también como un medio para contribuir al desarrollo de la sociedad. La apreciación de la educación coincide con la tradición de res- peto a la gente educada, especialmente a la dedicada a la docencia.

Por consiguiente, la educación formal se convirtió en un campo de batalla para la supervivencia y el éxito del individuo. Esto se debe a que, comparada con otras áreas de la vida social y política, la educación es un contexto donde es posible enfocarse en el desarrollo de los seres humanos. Tal característica ha sido acentuada por la percepción de que en el área educativa la gente tiene autonomía, aunque limitada. Como consecuencia, la familia es la unidad de combate y los hijos son los principales combatientes. Bajo esta perspectiva, los grupos de clase son flexibles en su estructura y, en momentos críticos, existe la tendencia a proteger los intereses individuales (o familiares) y no de grupo.

El Estado participa en esta guerra educativa a través de las políticas implementadas con las que a veces trata de reducir la demanda y otras veces alienta a la gente a luchar por una mejor educación. Con base en el anterior racionamiento, Weidman y Park alientan a futuros investigadores y políticos responsables de la educación coreana a facilitar un continuo desarrollo sin ignorar el contexto coreano caracterizado por la falta de recursos naturales, una alta densidad poblacional, una estructura social reprimida por guerras y dominación colonial, un rol preponderante de la educación en el desarrollo nacional y la remarcada voluntad de los padres de familia de sacrificarse por sus hijos. ${ }^{6}$

Más recientemente, Bong Gun Chung reconoció varias fallas en el sistema educativo con manifestaciones como el sistema de acreditación de profesores como un mecanismo de control de calidad en la educación, el cual se encuentra aún en una fase primaria. Chung menciona que la estructura burocrática centralizada impide iniciativas provenientes de individuos tanto en las escuelas públicas como privadas. La cultura estudiantil, que una vez estuvo inclinada hacia el activismo político radical y hacia un movimiento de democratización, ahora se encuentra imbuida en un 
consumismo de masas, lo que debilita la importancia de las actividades académicas. Aún más, el esfuerzo por conservar una competitividad a nivel mundial en un mercado globalizado y las políticas educativas gubernamentales han continuado acordes con los intereses económicos que esto exige. Los sistemas legales y las prácticas burocráticas siguen orientados hacia un control tanto sobre las universidades públicas como privadas. ${ }^{7}$

La reforma educativa llevada a cabo por el presidente Kim Young Sam en 1994 originó nuevas ideas y principios. La comisión presidencial encargada de llevar a cabo dicha reforma sintetizó las sugerencias hechas en 1985, cuando fue formado el consejo presidencial para la reforma educativa.

El gobierno de Kim Dae-Jung tomó un nuevo rumbo y se revisaron las sugerencias que se habían estado haciendo por varios años. Siete medidas principales fueron realizadas: la diversificación en los asuntos académicos a la vez que se favoreció su autonomía; el establecimiento de límites máximos de admisiones con la intención de conservar la oferta de mano de obra a un nivel estable; la abolición de la necesidad de aprobación gubernamental para crear universidades, especialmente las dedicadas a los programas de diseño, información y tecnología en comunicaciones; una innovación en la gerencia de profesores universitarios, facilitando así la inclusión de profesores extranjeros; la categorización de universidades: las que se dedican a la investigación y las que se enfocan a la docencia; el reconocimiento de las universidades que consideren los consejos de empresas comerciales a través de incentivos monetarios; y la creación del "Proyecto 'Brain Korea' para el siglo XXI”, mediante el cual se dan incentivos a la investigación de vanguardia, especialmente en ciencias e ingenierías (excluye explícitamente las áreas de artes, humanidades y ciencias sociales ${ }^{8}$ ).

Dichas iniciativas han encontrado oposición por parte del público, como lo hemos visto antes y en otros períodos. Los argumentos de oposición siguen denunciando una ex- clusión de las clases menos privilegiadas. La oposición reclama también que la educación universitaria no debe ser gobernada por las leyes del mercado. Las reformas han sido calificadas como neoliberales; sin embargo, vemos que aún hay un gran control gubernamental, por lo que esas acusaciones deben ser revisadas en este sentido. La manera en que las reformas han sido hechas es reforzada con leyes como el Acta de Educación Superior (The Higher Education Act), el Acta para Empleado en Educación Pública (The Public Educational Employee Act) y el Acta de Escuelas Privadas (The Private Schools Act). Estas leyes y reglamentos, denuncia Chung, ayudan a resolver conflictos entre la gente involucrada en el proceso educativo, pero también impiden el desarrollo de iniciativas en aras de conservar determinada igualdad e imparcialidad.

El Ministerio de Educación Coreano sigue siendo dominado por las presiones de otros ministerios con mayor poder sobre los recursos financieros nacionales y por el control general de la coordinación de las políticas gubernamentales. Como presión adicional, los grupos investigadores de apoyo educativo, como el Instituto de Desarrollo Coreano (KEDI, por sus siglas en inglés), favorece los intereses de mercado en el sector educativo. Otro factor con gran influencia es el hecho de que los profesores e investigadores encargados de orientar la reforma presentan una gran influencia por parte de las tendencias anglosajonas, pues ellos mismos han recibido entrenamiento en Estados Unidos o Inglaterra. Esto reduce la capacidad de considerar factores endógenos. ${ }^{9}$

A pesar de la continuidad en el conflicto entre los intereses gubernamentales y del público, la democratización de los noventa ha creado un nuevo ambiente comparado con los patrones observados durante los sesenta. Actualmente, la prensa, determinados grupos de interés, las organizaciones no gubernamentales y el congreso bipartisano ejercen más influencia sobre las decisiones gubernamentales. Ahora la reputación y el reconocimiento de los 
oficiales de altas esferas son menos válidos que antes. Aunado a esto, la crisis económica de 1997 ha sido reconocida como un resultado directo de la intervención exagerada del gobierno. Además, el sector privado, aunque esté siendo reformado internamente, tiene también gran influencia pues la reforma educativa necesita el apoyo del sector privado para ser justificada. ${ }^{10}$

Recientemente, los intelectuales han hecho hincapié en la influencia de la economía global basada en el poder del conocimiento y ya no tanto en el capital financiero o humano. El conocimiento o capacidad de manejo de información ha sido identificado como un recurso preponderante en el desempeño tanto de las naciones como de los individuos. De acuerdo con esto, se debe reconocer que la estructura burocrática actual, no solamente coreana sino de otros muchos países, es incongruente con la necesidad de crear y manejar información necesaria en un mundo posmoderno. Así, las reformas hechas de ahora en adelante tendrán que ser articuladas de una manera innovadora, pues el Ministerio de Educación no puede ser el único responsable de las políticas educativas, así como tampoco la sociedad. ${ }^{11}$

Las iniciativas del actual gobierno, bajo la presidencia de Roh Moo-hyun, han presentado nuevas reformas entre las que se incluye el Sistema de Desarrollo de Recursos Humanos (Human Resources Development System), el cual incluye al Ministerio de Educación y al ya existente Desarrollo de Recursos Humanos (Human Resources Development). Tal reestructuración se hizo en marzo de 2004, y ha sido justificada como un esfuerzo para asegurar la eficiencia de las nuevas políticas educativas. El gobierno explícitamente ha insistido que esta y otras reformas tienen como objetivo principal el refuerzo en la competitividad nacional ante todo. ${ }^{12}$

Las iniciativas del gobierno actual hacen evidente la continuidad en los esfuerzos oficiales por coordinar intereses económicos con el desarrollo educativo. Sin embargo, aún fal- ta un mayor enfoque en lo que Weydman y Park han propuesto, pues las características endógenas de la población coreana presentan variables que aparentemente no han sido consideradas en profundidad, no por falta de esfuerzos sino, como ya se mencionó, porque hay una clara inclinación en evaluar el escenario coreano desde una perspectiva internacional.

El Instituto de Investigación para la Educación y Entrenamiento Vocacional de Corea (KRIVET, por sus siglas en inglés) ha sugerido medidas para superar problemas de percepción en la sociedad, como la exclusividad y elitismo en la impartición de la educación tanto primaria, secundaria y superior. ${ }^{13}$ Esto es un claro esfuerzo por ganar el apoyo público.

Con este trabajo he tratado de mostrar una imagen precisa que clarifica la relación entre el desarrollo educativo y el desarrollo económico coreanos. Sin embargo, la intervención gubernamental en la educación en pro del desarrollo nacional ha sido evidentemente menos efectiva que en otras áreas. Asimismo, factores contradictorios como la falta de reconocimiento y validación de las uniones laborales para proteger los derechos de los maestros, constituyen otra variable a considerar en el estudio del desarrollo nacional coreano, entre otros. Existe la tendencia a presuponer que un desarrollo político democrático es creado donde se ha dado un desarrollo económico.

En el caso de Corea del Sur, el desarrollo económico no ha traído consigo necesariamente un avance paralelo hacia una sociedad democrática. Sin embargo, cabe decir que en la actualidad se están haciendo esfuerzos por coordinar ambos desarrollos por parte del gobierno y de la sociedad coreana, la cual también resalta su gran conciencia nacional.

\section{Notas}

1 Paik Hun-ki. "The Korean Social Structure and its implications for Education", en Aspects of Social Change in Korea. Ed. C.I. Eugene Kim. The Korea Research and Publications, Inc. Kalamazoo, Michigan. 1968, pág. 9. 
2 Referencia hecha por Don Adams y Esther E. Gottlieb, en Education and Social Change in Korea. Garland Publishing Inc., 1993, pág. 176.

3 Adams y Gottlieb, op. cit.

4 Ibid., pp. 178- 179.

5 Ibid., pp. 180-182.

6 John C. Weidman y Namgi Park. Higher Education in Korea, Tradition and Adaptation. Falmer Press, 2000.

7 Bong Gun Chung. The University Reform in Korea: a deregulation to be deregulated? Presentación hecha para la Segunda Conferencia en Investigación Educativa, 2001, Seoul National University, Seúl, Corea.

8 Ibid., pp. 4-7.

9 Ibid., p. 10.

10 Ibid., pp. 10-11.

11 Ibid., pp. 11-12.

12 http://www.moe.go.kr/en.

13 Idem.

\section{Fuentes}

Adams, Don y Esther E. Gottlieb (1993) Education and social change in Korea. Nueva York: Garland Pub.

Chung, Bong Gun (2001)The University Reform in Korea: a deregulation to be deregulated? Presentación hecha para la
Segunda Conferencia en Investigación Educativa, Seoul National University, Seúl, Corea.

Kim, Jongchol (1985) Education and development: some essays and thoughts on Korean education. Seúl: Seoul National University Press.

Kim, Ransoo (1984) Korean education: in research perspectives. Seúl: Jong-Gak.

Paik Hun-ki (1968) "The Korean Social Structure and its implications for Education", enAspects of Social Change in Korea. Ed. C.I. Eugene Kim. The Korea Research and Publications Inc. Kalamazoo, Michigan.

Seth, Michael J. (2002) Education fever: society, politics, and the pursuit of schooling in South Korea. Honolulu: University of Hawai'i Press and Center for Korean Studies, University of Hawai'i.

Weidman, John C. y Namgi Park (2000) Higher education in Korea: tradition and adaptation. Nueva York: Falmer Press.

http://www.moe.go.kr/en $7:$
The series Pacific Rim: Globalization, Regionalization, and Domestic Trajectories is composed of four volumes, under the general supervision of Melba Falck and Arturo Santa-Cruz

Volume 1. Globalization, Regionalization and Domestic Trajectories in the Pacific Rim: The Economic Impact. Edited by Melba Falck and Arturo Santa-Cruz

Volume 2. Globalization, Regionalization and Social Change in the Pacific Rim. Edited by Kate Barclay and Wayne Peake

Volume 3. Regionalization, Marketization and Political Change in the Pacific Rim. Edited by James Goodman

Volume 4. Globalization and Regionalization: Views from the Pacific Rim. Edited by Stephanie Lawson.

\section{Volume 1. Globalization, Regionalization and Domestic Trajectories in the Pacific Rim: The Economic I mpact}

\author{
Melba Falck \\ Arturo Santa-Cruz (editors) \\ Primera edición, 2004 \\ Universidad de Guadalajara \\ Precio estudiantes $\$ 112.00$ \\ Precio público $\$ 205.00$
}

De venta en: librería el Kiosco, Centro Universitario de Ciencias Sociales y Humanidades, Av. Alcalde esquina Av. de los Mestros, edificio G, planta baja, Guadalajara, Jalisco 